Published in "Diagnostic Microbiology and Infectious Disease

81(4): 264-268, 2015"

which should be cited to refer to this work.

\title{
Clonal distribution of multidrug-resistant Enterobacter cloacae
}

\author{
Delphine Girlich ${ }^{\mathrm{a}}$, Laurent Poirel ${ }^{\mathrm{a}, \mathrm{b}}$, Patrice Nordmann ${ }^{\mathrm{a}, \mathrm{b}, \mathrm{c}, *}$ \\ a INSERM U914 "Emerging Resistance to Antibiotics", K.-Bicêtre, France \\ ${ }^{\mathrm{b}}$ Medical and Molecular Microbiology, Department of Medicine, Faculty of Science, University of Fribourg, Fribourg, Switzerland

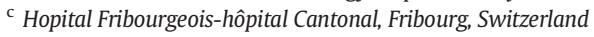

\begin{abstract}
A multilocus sequence typing (MLST) scheme including 7 housekeeping genes was used to evaluate whether the current spread of multidrug-resistant Enterobacter cloacae isolates worldwide might be associated to specific successful clones. Fifty E. cloacae clinical isolates of worldwide origin, with various $\beta$-lactamase content, and recovered at different periods of time were studied. Forty-four sequence types were identified, highlighting a high clonal diversity with 3 main lineages. This study revealed that a precise identification of the isolates by sequencing of the chromosomal ampC gene of E. cloacae would provide a significant added value to improve the reliability of the MLST scheme.
\end{abstract}

\section{Introduction}

Although Enterobacter cloacae is an enterobacterial species that possesses several naturally occurring resistance mechanisms, acquired multidrug resistance is increasingly observed. Resistance to $\beta$-lactams in that species may be related to overexpression of chromosomal ampC $\beta$-lactamase genes, acquisition of plasmid-mediated extendedspectrum $\beta$-lactamase (ESBL), or carbapenemase genes, those latter genes encoding KPC type; OXA-48 type; or metallo- $\beta$-lactamases of the VIM-, IMP-, and NDM-1 types (Girlich et al., 2014; Ikonomidis et al., 2007; Novak et al., 2014; Pasanen et al., 2014; Pestourie et al., 2014; Poirel et al., 2014). Identifying clonal spread of multidrugresistant isolates recovered from geographically distant locations remains difficult, since data are being obtained mainly by using the pulsed-field gel electrophoresis technique, which provides heterogeneous and noncomparable data. By contrast, multilocus sequence typing (MLST) provides interlaboratory comparison of epidemiological data. We have used here a recently described MLST scheme including 7 housekeeping genes (Miyoshi-Akiyama et al., 2013) in order to perform an epidemiological comparison of 50 multidrug-resistant $E$. cloacae. This study focuses on multidrug-resistant isolates and, in particular, those producing the NDM- 1 or OXA-48 carbapenemases and the ESBL CTX-M-15. The aim was to evaluate i) whether some specific sequence types (STs) with a peculiar $\beta$-lactamase content might be disseminated among various countries and ii) whether ESBL- and carbapenemaseproducing isolates might correspond to the same strain backgrounds as those of the ESBL-positive but carbapenemase-negative isolates.

\footnotetext{
* Corresponding author. Tel.: +41-26-300-9581.

E-mail address: patrice.nordmann@unifr.ch (P. Nordmann).
}

\section{Methods}

\subsection{Clinical isolates}

Fifty E. cloacae clinical isolates of worldwide origin and recovered at different periods of time (from 1994 to 2013) were included. They were randomly selected from our international collection, including isolates from India, Lebanon, France, Vietnam, Morocco, Turkey, and Algeria. They have been characterized for their $\beta$-lactamase content at the molecular level. The isolates were either wild-type strains $(n=3)$, AmpC overproducers $(\mathrm{n}=10)$, ESBL producers of the CTX-M-15 type $(\mathrm{n}=16)$, and carbapenemase producers $(n=24)$ (Table 1$)$. Isolates were identified by the API20E biochemical test (bioMérieux, Marcy l'Etoile, France).

\subsection{MLST and phylogenetic analysis}

MLST primers targeted 7 housekeeping genes (dnaA, fusA, gyrB, leuS, pyrG, rplB, and $r p o B$ ) with PCR conditions, as recommended (MiyoshiAkiyama et al., 2013). Purified PCR products were sequenced using an ABI3130 apparatus (Applied Biosystems, Life Technologies SAS, Saint Aubin, France). Concatenated sequences of the 7 DNA fragments (3501 nucleotides in total) were obtained from the Web site (http:// pubmlst.org/ecloacae/) and compared using the Unweighted Pair Group Method with Arithmetic Mean (UPGMA) method. A phylogenic tree was inferred by bootstrap phylogenetic inference using Molecular Evolutionary Genetics Analysis Version 6.0. software (MEGA6) (Tamura et al., 2013). These sequences were compared with 3 concatenated reference sequences (ST2, ST3, and ST9), corresponding to the 3 main clades of ST for E. cloacae isolates, as reported by Miyoshi-Akiyama et al. (2013). Searches of the GenBank databases were carried out using the NCBI BLASTn option (www.ncbi.nlm.nih. 
Table 1

E. cloacae isolates used in this study.

\begin{tabular}{|c|c|c|c|c|c|c|c|c|c|c|c|c|}
\hline Isolate & B-lactamase content & $\begin{array}{r}\text { Year : } \\
\text { of }\end{array}$ & $\begin{array}{l}\text { and country } \\
\text { isolation }\end{array}$ & ST & $d n a A$ & fus $A$ & gyr & leuS & $p y r G$ & $r p l B$ & $r p o B$ & lineage \\
\hline 5434 & wild type & 2011 & France & 237 & 4 & 6 & 4 & 6 & 37 & 36 & 25 & 1 \\
\hline 7725 & wild type & 2011 & France & 238 & 58 & 40 & 81 & 9 & 79 & 37 & 38 & 3 \\
\hline 7746 & wild type & 2011 & France & 247 & 58 & 61 & 99 & 102 & 97 & 6 & 55 & 7 \\
\hline KAU & overexpressed AmpC & 2012 & France & 108 & 68 & 8 & 75 & 63 & 65 & 34 & 35 & 1 \\
\hline VIL & overexpressed AmpC & 2012 & France & $\mathbf{5 0}$ & 4 & 4 & 4 & 6 & 37 & 4 & 25 & 1 \\
\hline BRE & overexpressed AmpC & 2012 & France & 239 & 58 & 37 & 4 & 6 & 4 & 4 & 25 & 1 \\
\hline COUP & overexpressed AmpC + TEM-121 & 2012 & France & 248 & 92 & 60 & 100 & 99 & 9 & 42 & 56 & $-^{a}$ \\
\hline BALD & overexpressed AmpC + TEM-2 & 2012 & France & 240 & 8 & 9 & 6 & 9 & 9 & 4 & 8 & 3 \\
\hline CON & overexpressed AmpC & 2012 & France & 241 & 4 & 4 & 37 & 6 & 81 & 4 & 25 & 1 \\
\hline AZA & overexpressed AmpC & 2012 & France & 249 & 86 & 25 & 96 & 100 & 100 & 14 & 57 & 8 \\
\hline KHA & СТX-M-15 & 2008 & France & 198 & 68 & 8 & 75 & 63 & 65 & 34 & 6 & 1 \\
\hline FOU & CTX-M-15 & 2008 & France & 78 & 8 & 9 & 6 & 9 & 9 & 6 & 6 & 3 \\
\hline 3 & CTX-M-15 (ArmA) & 2008 & India & 199 & 46 & 21 & 20 & 96 & 45 & 29 & 54 & 2 \\
\hline CAU & CTX-M-15 + TEM-1 & 2008 & France & 168 & 59 & 40 & 81 & 9 & 79 & 37 & 38 & 3 \\
\hline NAI & CTX-M-15 + SHV-12 & 2008 & France & 196 & 8 & 33 & 86 & 9 & 9 & 6 & 8 & 3 \\
\hline DAS & CTX-M-15 + TEM-1 & 2008 & France & 201 & 53 & 35 & 20 & 44 & 45 & 38 & 8 & 2 \\
\hline KER & CTX-M-15 & 2009 & France & 202 & 53 & 35 & 20 & 44 & 45 & 29 & 32 & 2 \\
\hline $\mathrm{HOF}$ & CTX-M-15 & 2009 & France & 195 & 53 & 35 & 20 & 44 & 45 & 4 & 53 & 2 \\
\hline RIV & CTX-M-15 & 2009 & France & 251 & 85 & 63 & 101 & 103 & 96 & 6 & 6 & 8 \\
\hline $\mathrm{CON}$ & CTX-M-15 & 2009 & France & $114^{b}$ & 53 & 35 & 20 & 44 & 45 & 4 & 6 & 2 \\
\hline ZER & CTX-M-15 + TEM-1 & 2010 & France & 114 & 53 & 35 & 20 & 44 & 45 & 4 & 6 & 2 \\
\hline BAL & CTX-M-15 & 2010 & France & 114 & 53 & 35 & 20 & 44 & 45 & 4 & 6 & 2 \\
\hline BAR & CTX-M-15 + TEM-1 & 2010 & France & 207 & 53 & 35 & 20 & 45 & 45 & 4 & 6 & 2 \\
\hline SAM & CTX-M-15 + TEM-1 & 2011 & France & 114 & 53 & 35 & 20 & 44 & 45 & 4 & 6 & 2 \\
\hline CAR & CTX-M-15 & 2011 & France & 236 & 59 & 64 & 81 & 9 & 79 & 37 & 6 & 8 \\
\hline BRA & CTX-M-15 + TEM-1 + SHV-28 & 2011 & France & 200 & 74 & 20 & 20 & 65 & 45 & 4 & 32 & 2 \\
\hline NOR & NMC-A & 1994 & France & 250 & 84 & 62 & 95 & 98 & 94 & 43 & 52 & 6 \\
\hline KAR & VIM-1 + SHV-70 & 2011 & France & 229 & 87 & 16 & 25 & 97 & 22 & 9 & 15 & 6 \\
\hline KOW3 & VIM-4 + CTX-M-15 + TEM-1 + SHV-31 & 2011 & Kuwait & 203 & 4 & 4 & 20 & 6 & 92 & 30 & 6 & 1 \\
\hline TWA & IMP-8 & 2011 & Taiwan & 194 & 11 & 6 & 4 & 13 & 39 & 4 & 9 & 1 \\
\hline TAW & IMP-8 + SHV-12 & 2011 & Taiwan & 204 & 4 & 4 & 4 & 6 & 95 & 4 & 6 & 1 \\
\hline RAZ & NDM-1 & 2012 & Vietnam & 193 & 49 & 20 & 7 & 44 & 90 & 24 & 32 & 2 \\
\hline PAY & NDM-1 & 2012 & France & 230 & 49 & 20 & 74 & 44 & 90 & 24 & 32 & 2 \\
\hline SEN & NDM-1 & 2012 & France & 205 & 4 & 6 & 4 & 61 & 39 & 4 & 25 & 1 \\
\hline BOQ & NDM-1 & 2012 & France & 231 & 46 & 20 & 20 & 96 & 45 & 29 & 54 & 2 \\
\hline $\mathrm{ABA}$ & NDM-1 + CTX-M-15 + TEM-1 + OXA-1 & 2013 & France & 200 & 74 & 20 & 20 & 65 & 45 & 4 & 32 & 2 \\
\hline GAT & NDM-1 + CTX-M-15 + TEM-1 + OXA-1 & 2013 & France & 206 & 67 & 20 & 20 & 44 & 45 & 4 & 32 & 5 \\
\hline IR38 C & NDM-1 + CTX-M-15 & 2012 & India & 235 & 49 & 21 & 19 & 44 & 94 & 12 & 32 & 4 \\
\hline LIB & NDM-1 + CTX-M-15 & 2012 & Lebanon & 32 & 3 & 24 & 3 & 35 & 3 & 16 & 17 & 3 \\
\hline MAR17 & OXA-48 + TEM-1 + CTX-M-9 & 2009 & Morocco & 197 & 67 & 21 & 74 & 95 & 45 & 35 & 6 & 2 \\
\hline MAR18 & OXA-48 + TEM-1 + SHV-12 + CTX-M-9 & 2009 & Morocco & 197 & 67 & 21 & 74 & 95 & 45 & 35 & 6 & 2 \\
\hline MAR19 & OXA-48 + TEM-1 + CTX-M-15 & 2009 & Morocco & 192 & 46 & 20 & 74 & 44 & 45 & 24 & 42 & 2 \\
\hline BOU & OXA-48 + TEM-1 + CTX-M-15 + OXA-1 & 2010 & Morocco & 245 & 91 & 59 & 19 & 44 & 99 & 4 & 32 & 2 \\
\hline TUR & OXA-48 + SHV-5 & 2010 & Turkey & 120 & 46 & 20 & 20 & 44 & 45 & 29 & 6 & 2 \\
\hline MAR20 & OXA-48 + TEM-1 + SHV-12 & 2011 & Morocco & 190 & 9 & 4 & 15 & 6 & 37 & 4 & 9 & 1 \\
\hline 501 & OXA-48 + TEM-1 + CTX-M-15 + OXA-1 & 2011 & Morocco & 244 & 90 & 20 & 19 & 44 & 45 & 4 & 6 & 2 \\
\hline $\mathrm{BEU}$ & $\begin{array}{l}\text { OXA-48 + TEM-1 + SHV-12 + CTX-M- } \\
15 \text { + DHA-1 + OXA-1 }\end{array}$ & 2011 & France & 182 & 49 & 20 & 19 & 44 & 90 & 24 & 32 & 2 \\
\hline ESS & OXA-48 + CTX-M-15 & 2011 & Algeria & 25 & 24 & 14 & 43 & 52 & 27 & 18 & 21 & 6 \\
\hline $\mathrm{AZZ}$ & OXA-48 + TEM-1 + CTX-M-15 + OXA-1 & 2011 & Morocco & 246 & 67 & 59 & 19 & 44 & 99 & 4 & 32 & 2 \\
\hline DOV & OXA-48 + TEM-1 + CTX-M-15 + OXA-1 & 2011 & Morocco & 114 & 53 & 35 & 20 & 44 & 45 & 4 & 6 & 2 \\
\hline
\end{tabular}

${ }^{a}$ Isolates of ST 25, 229, 249, and 250 have been identified as E. asburiae; isolate of ST248 has been identified as E. aerogenes and should, therefore, be excluded from this MLST scheme if strictly restricted for E. cloacae isolates.

${ }^{\mathrm{b}}$ E. cloacae with ST114 are indicated with a gray shadow. 


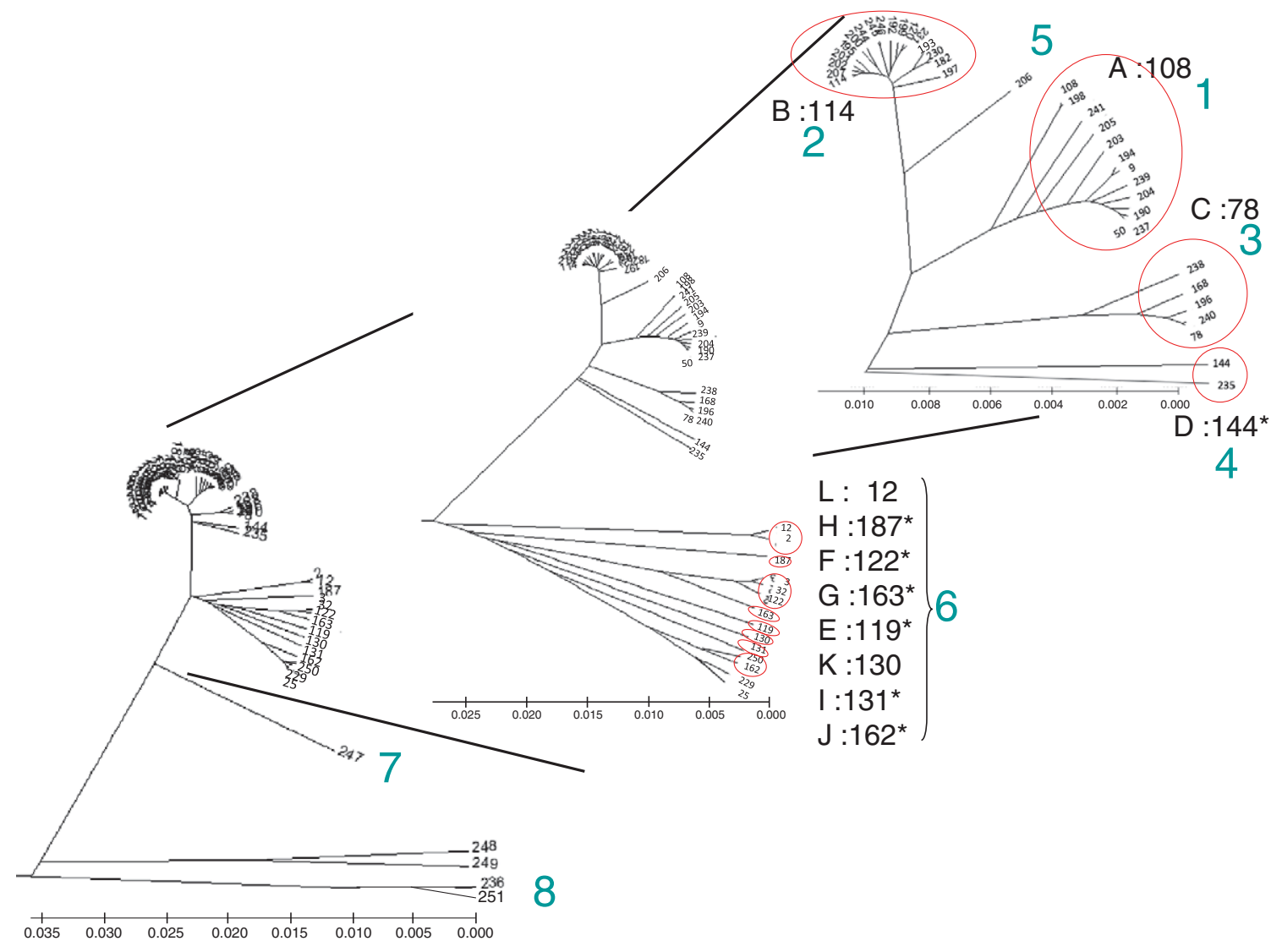

Fig. 1. Unrooted tree of concatenated sequences from combinations of 7 MLST loci. Phylogenetic analysis of 44 distinct ST from fifty E. cloacae isolates, 3 reference ST (ST2, ST3, and ST9), from Miyoshi-Akiyama et al. (2013) and STs from the study of Izdebski et al. (2015) as representatives of each cluster (letters a to L) previously reported. The eight lineages identified in this study are indicated with numbers (Girlich et al., 2014; Grundmann et al., 2001; Ikonomidis et al., 2007; Izdebski et al., 2015; Miyoshi-Akiyama et al., 2013; Novak et al., 2014; Pasanen et al., 2014; Pestourie et al., 2014). The STs not identified in our study were marked with an asterisk. The tree was inferred by bootstrap phylogenetic inference using MEGA6 software.

gov), and precise identification of the species was carried out using the rpoB sequence on the bioinformatics database leBIBI (umr5558-sudstr1.univ-lyon1.fr/lebibi/lebibi.cgi). The clonal diversity index and confidence intervals (CIs) were calculated according to Grundmann et al. (2001).

\subsection{Chromosomal ampC and phylogenetic analysis}

Considering that E. cloacae is a complex of several species and subspecies, the $a m p C$ gene encoding the intrinsic $A m p C \beta$-lactamase of the species E. cloacae was amplified and sequenced with specific primers, namely, ECA (5'-CCCTTTGCTGCGCCCTGC-3') and ECB (5'-TGCCGCCTCAACGCGTGC-3'). Since amplifications failed for isolates belonging to ST248 and ST249, PCR with primers specific for the intrinsic ampC genes of other Enterobacter species were also attempted. The ampC gene of Enterobacter aerogenes was amplified with primers EaerA (5'-CAATCAGACCATCACGCCGT- $3^{\prime}$ ) and EaerB (5'-AGCATCACGATACCGAGATCC-3') and that of Enterobacter asburiae with primers EasbA (5'-GGAACGTACCGTTACGCCGC-3') and EasbB (5'GCCGGTTTTATGGACCCAGG-3'). Sequences of the ampC genes (790-bp long) were compared using the UPGMA method. A phylogenic tree was inferred by bootstrap phylogenetic inference using MEGA6 (Tamura et al., 2013).

\subsection{Nucleotide sequence accession numbers}

The ampC gene sequences of isolates 7746 of ST247, RIV of ST251, AZA of ST249, NOR of ST250, and ESS of ST25 appear in the GenBank database with accession numbers KM985624 to KM985628.

\section{Results and discussion}

\subsection{MLST and phylogenetic analysis}

Forty-four STs were identified among the 50 E. cloacae isolates, highlighting a high clonal diversity (Table 1). Out of 44 distinct ST identified, 39 (88.6\%) corresponded to novel ones. The clonal diversity index was $93.7 \%$ with a $\mathrm{Cl}$ value of $92.5-94.9 \%$, indicating a high genetic diversity. Phylogenetic analysis revealed little association either to the antibiotic resistance gene content or to the geographic origin of the isolates (Table 1 and Fig. 1). This result is consistent with results from Izdebski et al. (2015), who reported very recently a clonal diversity of $97.5 \%$ among expanded-spectrum cephalosporin-resistant $E$. cloacae isolates from Europe and Israel (Izdebski et al., 2015). The discriminatory ability of the different loci, measured as the number of alleles obtained for each gene, varied from 17 (for $r p o B$ and $r p l B$ ) to 21 (for $d n a A$ ) (Table 1). This result differs from that obtained by Miyoshi-Akiyama et al. (2013), reporting the leuS and pyrG alleles as the most polymorphic ones. The result of the nucleotide diversity analysis within the sample, performed on alleles of each MLST gene, demonstrated significant diversity in accordance with the data from Miyoshi-Akiyama et al. (2013) and Izdebski et al. (2015).

The most frequently identified alleles were dnaA-53 (9 isolates), fusA20 (10 isolates), gyrB-20 (16 isolates), leuS-44 (18 isolates), pyrG-45 (19 isolates), $r p l B-4$ ( 21 isolates), and $r p o B-6$ (15 isolates) (Table 1$)$. Even though the combination of all these alleles in a given ST profile has not been reported yet, it is very close to that of the most frequently identified ST of this study (ST114), differing only by a single locus (fusA) (Table 1). 


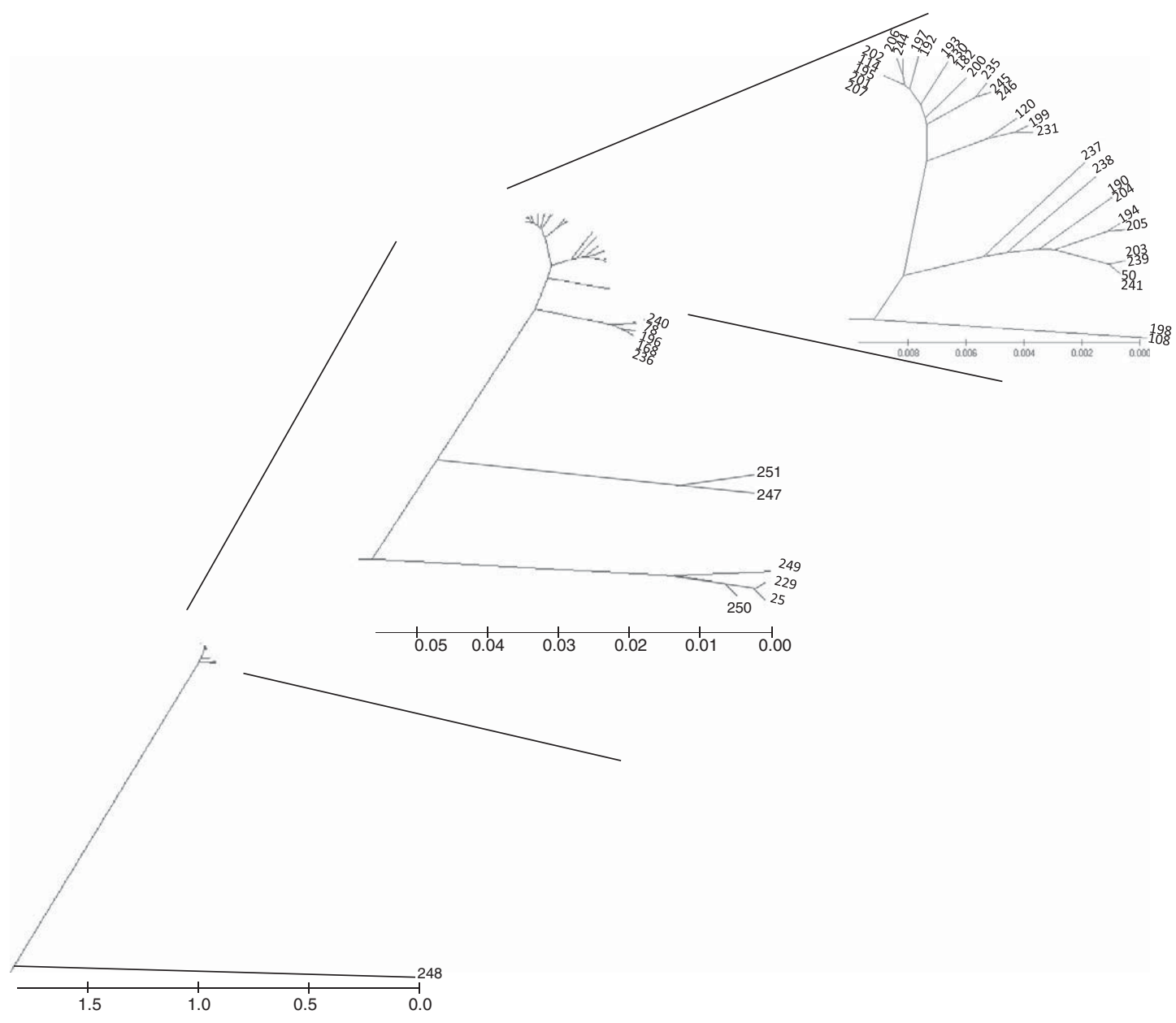

Fig. 2. Unrooted tree of chromosomal ampC sequences from the 50 Enterobacter isolates. Phylogenetic analysis of the $50 \mathrm{E}$. cloacae isolates used in this study after sequencing of their chromosomal ampC gene. The tree was inferred by bootstrap phylogenetic inference using MEGA6 software. The isolate of ST248, identified as an E. aerogenes isolate, and ST25, ST229, ST249, and ST250, identified as E. asburiae isolates after sequencing of the ampC genes, should be excluded from MLST lineages.

The global phylogeny revealed 8 lineages. Three major lineages (namely, 1,2 , and 3) encompassed most $E$. cloacae isolates, represented by ST78 like (group 3), ST108 like (group 1), and ST114 like (group 2) (Fig. 1). Each of these lineages contained concatenated sequences showing $>99 \%$ nucleotide identity and shared ca. $98 \%$ of nucleotide identity with each other. In order to compare with the 3 main clades reported by Miyoshi-Akiyama et al. (2013), concatenated sequences of ST2, ST3, and ST9 (respectively, corresponding to clades 1,2 , and 3) were included in our phylogenetic analysis. Surprisingly, the 8 lineages identified in our study did not correlate with the 3 clades previously reported. For instance, ST2 and ST3 from Miyoshi-Akiyama et al. (2013) were included in the same lineage containing ST32 and showed ca. 94\% nucleotide identity with the ST114 lineage (Fig. 1).

The phylogenetic analysis of Izdebski et al. (2015) performed on 195 E. cloacae resistant to expanded spectrum cephalosporins and collected across Europe and Israel distinguished 80 STs and contained 12 clusters of very related STs. Comparing with those data, we were able to redefine our lineages accordingly, aiming to make both studies corremating as much as possible. The phylogenetic analysis was, therefore, completed by including the 12 STs representing the 12 different clusters as defined by Izdebski et al. (2015). The first 4 lineages could be well correlated with those (A to D) from Izdebski et al. (2015), but some STs that had not been previously identified could not be related to any of the previously described clusters.

\subsection{Chromosomal ampC and phylogenetic analysis}

Sequencing of the $a m p C$ genes showed a lower diversity than that obtained with the 7 housekeeping genes used for the MLST. The phylogenetic tree obtained with the $a m p C$ sequences showed only 5 main lineages and differed from that obtained with the concatenated sequences (Fig. 2). Noticeably, isolates with the same ST harbored the same ampC sequence (ST114, ST197, and ST200). However, the ampC genes of ST247 and ST251 isolates, those of ST25, ST229, ST249, and ST250 isolates, and that of the ST248 isolate significantly differed from those of all other isolates (Fig. 2). The ampC gene of the ST247 isolate shared the highest identity with that of ST251, with a value of $97.3 \%$ and a maximum of $89.4 \%$ nucleotide identity with that of other $E$. cloacae isolates.

Since the amplification of the $a m p C$ gene from a single isolate belonging to ST248 failed, primers EaerA and EaerB specific for the E. aerogenes $a m p C$ gene were used and gave a positive result. Sequence analysis iden-

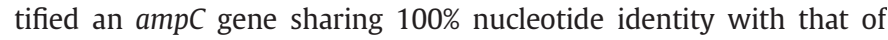
E. aerogenes EA1509E (Preston et al., 2000), and no more than $73.4 \%$ nucleotide identity with the $a m p C$ genes identified from $E$. cloacae. As suggested in Fig. 1, this ST248 isolate might, therefore, be excluded from the MLST lineage, considering that this isolate was initially misidentified. Indeed, the closest rpoB sequence from that of the ST248 isolate was that of $E$. aerogenes CP002824. This indicates that the fragment length of the $r p o B$ gene used in the MSLT scheme might be too short for a precise identification of the species. By contrast, the use of the chromosomal ampC gene should be preferred for identification of the species.

In addition, since the amplification of the ampC gene from the ST249 isolate failed with primers specific for $E$. cloacae, primers EasbA and EasbB specific for the E. asburiae ampC gene were used and gave a positive result. Sequencing identified this $a m p C$ gene with $93 \%$ nucleotide identity compared to that of $E$. asburiae. Moreover, all others isolates grouped 
with that of ST249 in Fig. 2, e.g., ST25, ST229, and ST250, harbored chromosomal ampC genes with 98-99\% nucleotide identity with that of E. asburiae. All those isolates should, therefore, be excluded from the MLST scheme of E. cloacae species by further modifying the specificity of the typing (i.e., targeting other housekeeping genes, modifying primer locations).

\subsection{Clonal distribution of multiresistant E. cloacae isolates}

All these observations, although limited to a little number of isolates, suggest that there was no significant relationship between a specific $\beta$ lactam resistance profile and the major E. cloacae clonal groups (ST78, ST114, and ST108). Nevertheless, E. cloacae isolates belonging to ST114 were prevalent. Moreover, even though all bla $\mathrm{CTX}_{\mathrm{CM}-15}$-positive isolates did not all belong to ST114, most of them actually belonged to the clonal complex 114, with ST114 as the main representative ST (Table 1; Fig. 1).

\section{Conclusion}

We found here an important clonal diversity of multidrug-resistant E. cloacae isolates. No obvious link with a specific $\beta$-lactamase content or with a specific geographical origin could be evidenced. Nevertheless, the most prevalent ST identified was ST114. Further studies will be required to further evaluate the possible involvement of this clone in the dissemination of the $b a_{\mathrm{CTX}-\mathrm{M}-15}$ gene. Moreover, this study highlights some drawbacks of the current MLST scheme that may erroneously include non $E$. cloacae species and, therefore, bias the analysis. We believe that including the chromosomal $\operatorname{ampC}$ gene of $E$. cloacae would provide an added value to the reliability of the scheme.

\section{Funding}

This work was funded by the INSERM, France; by the University of Fribourg, Switzerland; and by grants from the European Community
(R-GNOSIS, FP7/HEALTH-F3-2011-282512, and MAGIC-BULLET, FP7/

HEALTH-F3-2001-278232).

\section{Transparency declarations}

None to declare.

\section{References}

Girlich D, Bouihat N, Poirel L, Benouda A, Nordmann P. High rate of faecal carriage of extended-spectrum $\beta$-lactamase and OXA-48 carbapenemase-producing Enterobacteriaceae at a university hospital in Morocco. Clin Microbiol Infect 2014;20:350-4.

Grundmann H, Hori S, Tanner G. Determining confidence intervals when measuring genetic diversity and the discriminatory abilities of typing methods for microorganisms. J Clin Microbiol 2001;39:4190-2.

Ikonomidis A, Spanakis N, Poulou A, Pournaras S, Markou F, Tsakris A. Emergence of carbapenem-resistant Enterobacter cloacae carrying VIM-4 metallo- $\beta$-lactamase and SHV-2a extended-spectrum $\beta$-lactamase in a conjugative plasmid. Microb Drug Resist 2007;13:221-6.

Izdebski R, Baraniak A, Herda M, Fiett J, Bonten MJ, Carmeli Y, et al. MLST reveals potential high-risk international clones of Enterobacter cloacae. J Antimicrob Chemother. 2015; 70:48-56.

Miyoshi-Akiyama T, Hayakawa K, Ohmagari N, Shimojima M, Kirikae T. Multilocus sequence typing (MLST) for characterization of Enterobacter cloacae. PLoS One 2013; 8:e66358.

Novak A, Goic-Barisic I, Tambic Andrasevic A, Butic I, Radic M, Jelic M, et al. Monoclonal outbreak of VIM-1-carbapenemase-producing Enterobacter cloacae in intensive care unit, university hospital centre split, Croatia. Microb Drug Resist 2014;20:399-403.

Pasanen T, Jalava J, Horsma J, Salo E, Pakarinen M, Tarkka E, et al. An outbreak of CTX-M15-producing Escherichia coli, Enterobacter cloacae, and Klebsiella in a children's hospital in Finland. Scand J Infect Dis 2014;46:225-30

Pestourie N, Garnier F, Barraud O, Bedu A, Ploy MC, Mounier M. Outbreak of AmpC Blactamase-hyper-producing Enterobacter cloacae in a neonatal intensive care unit in a French teaching hospital. Am J Infect Control 2014;42:456-8.

Poirel L, Yilmaz M, Istanbullu A, Arslan F, Mert A, Bernabeu S, et al. Spread of NDM-1producing Enterobacteriaceae in a neonatal intensive care unit in Istanbul, Turkey. Antimicrob Agents Chemother 2014;58:2929-33.

Preston KE, Radomski CC, Venezia RA. Nucleotide sequence of the chromosomal ampC gene of Enterobacter aerogenes. Antimicrob Agents Chemother 2000:44:3158-62.

Tamura K, Stecher G, Peterson D, Filipski A, Kumar S. MEGA6: Molecular Evolutionary Genetics Analysis Version 6.0. Mol Biol Evol 2013;30:2725-9. 